\title{
BUSINESS IS CHANGING: EXPERT AND PUBLIC OPINION ON THE FUTURE OF WORK AND ARTIFICIAL INTELLIGENCE THROUGH SOCIAL MEDIA RESEARCH
}

\author{
By \\ Ryan A. Cecchini \\ Honours Bachelor of Arts, University of Ottawa, 2017
}

\author{
A major research project \\ presented to Ryerson University \\ in partial fulfillment of the requirements for the degree of \\ Master of Professional Communication
}

Dr. Robert Clapperton

Toronto, Ontario, Canada, 2020

(C) Ryan A. Cecchini, 2020 


\section{Author's Declaration}

I hereby declare that I am the sole author of this MRP. This is a true copy of the MRP, including any required final revisions.

I authorize Ryerson University to lend this MRP to other institutions or individuals for the purpose of scholarly research.

I further authorize Ryerson University to reproduce this MRP by photocopying or by other means, in total or in part, at the request of other institutions or individuals for the purpose of scholarly research.

I understand that my MRP may be made electronically available to the public. 


\begin{abstract}
Big name organizations have proposed that AI will fundamentally transform the future of business by altering the skills that will be required to stay competitive in an epoch where routinejobs are automated and where workers in less-routine jobs are displaced from burgeoning advancements in AI. When it comes to assessing the impact of hazardous technologies, a growing body of literature suggests that experts and everyday people tend to perceive risks differently. Social media is a tool that allows individuals to engage with one another, and more recently, social media has become an influential means of consuming and disseminating news online. Reddit is a form of social media that combines both news consumption and social engagement. This major research project (MRP) examines online discourses from end-users on Reddit, from articles written by journalists, and from experts quoted in the media. Inductive content analysis reveals that topics of conversation about AI and the future of work centre around inequality and the economy. Apparent differences are observed between end-users, journalists, and experts, such as the high prevalence of dread on Reddit, which may suggest that most individuals, as opposed to those in the news media, think negatively about AI being introduced into the workplace — for reasons that revolve around the economy, inequality, or both.
\end{abstract}




\section{Acknowledgements}

I would like to thank my Supervisor Dr. Robert Clapperton for sharing his experience and knowledge. I would also like to thank my Second Reader Dr. Frauke Zeller for her valuable and constructive comments. Lastly, I would like to express my gratitude to Dr. Matthew Tiessen for his year-round counsel. 


\section{Dedication}

Mom, your support and patience helped make this possible. I am so grateful to have had you cheer me on every step of the way.

Dad, your encouraging words guided me throughout this tortuous journey, even when the paths forked.

Kyle, our friendship is an accolade I would not trade for the world. We have grown together, and we have learned so much from one another. You always listened.

Thank you all. 


\section{Table of Contents}

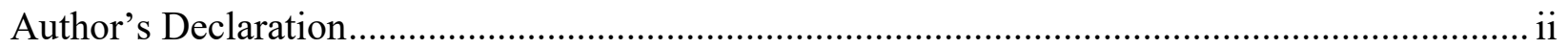

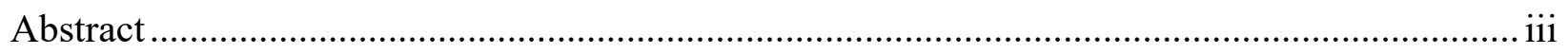

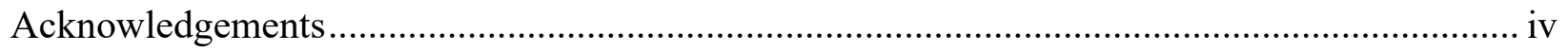

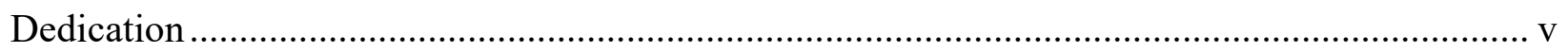

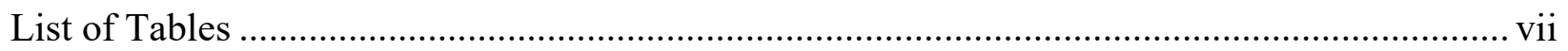

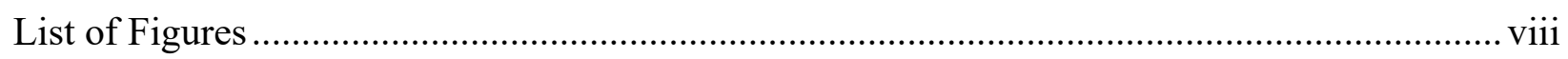

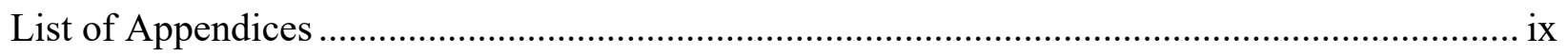

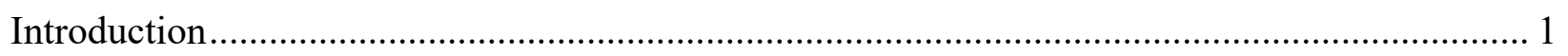

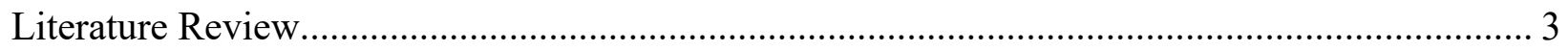

AI and the Future of Work....................................................................................... 3

Online Journalism and Social Media ........................................................................... 7

Public Opinion and Science Deliberation ................................................................... 9

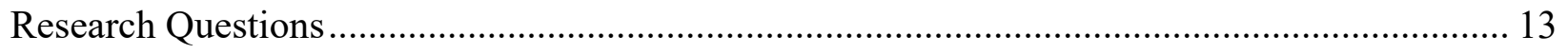

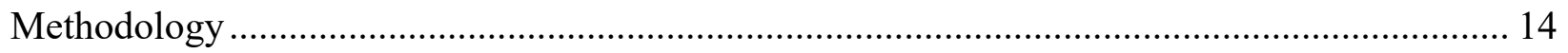

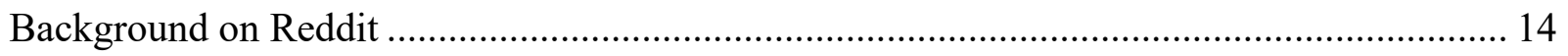

Methods and Data Extraction..................................................................................... 15

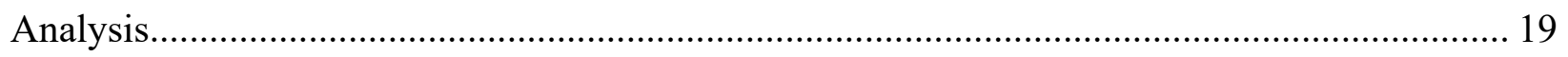

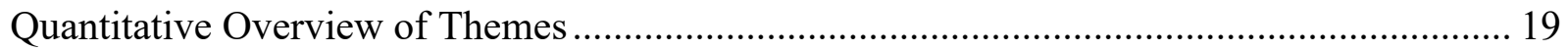

Qualitative Overview of Themes Analysis ............................................................................ 22

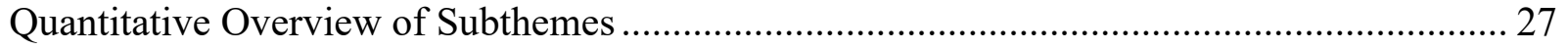

Qualitative Overview of Subthemes Analysis ........................................................... 32

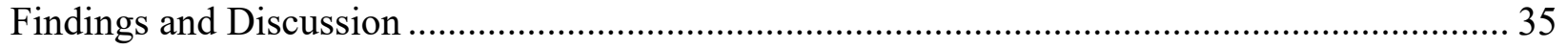

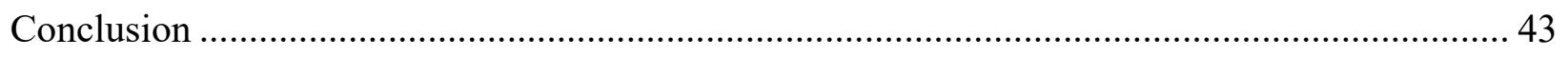

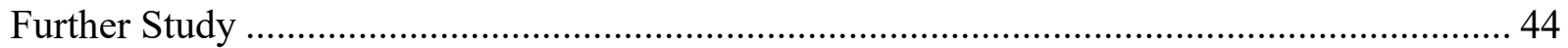

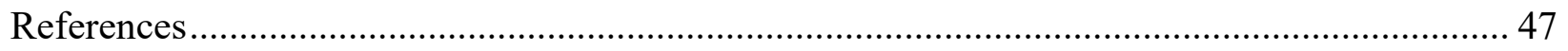




\section{List of Tables}

Table 1: Frequency Distribution of Themes for Overall Data ................................................ 20

Table 2: Frequency Distribution of Subthemes for Overall Data ........................................... 27

Table 3: Frequency Distribution of Subthemes by Unit of Analysis...................................... 30

Table 4: Frequency Distribution of Themes and Subthemes ................................................... 31 


\section{List of Figures}

Figure 1: Frequency of Themes in Journalists and Experts ................................................... 21 


\section{List of Appendices}

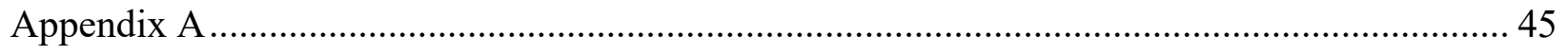

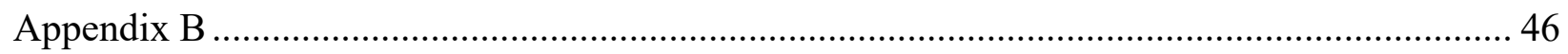




\section{Introduction}

Technological innovation is a notable aspect of modern and contemporary life, and in industry, the impact of technological innovation has been ubiquitous. Waves of mechanization have had lasting impacts on a variety of different industry sectors, and the introduction of new and improved technology has only gotten more rapid in the $21^{\text {st }}$ century. According to the World Economic Forum (WEF), this sort of exponential technological growth is indicative of a fourth industrial revolution because improved technologies are rapidly facilitating productivity and determining which skills will be in demand in the future (Schwab, 2016). Innovations in technology, such as the mass production of automobiles, have traditionally limited job automation to workers carrying out routine tasks (Autor, 2015). Recent technological advancements, however, such as AI-generated art, suggest that non-routine tasks could be similarly susceptible to labour displacement. AI aspires to simulate human faculties, and when those faculties enable AI to perform more dynamic job-related tasks, the range of occupations that could be automated widens.

Information about emerging technologies, not to mention AI, is readily communicated in the news media, and online social engagement has become a popular means of staying informed, demonstrated by the increasing number of individuals opting to use social media as a medium for news consumption (Shearer, 2018). Social news sites like Reddit are variations of social media that synergize news dissemination and social engagement by affording end-users the ability to share and discuss current affairs on the Web more efficiently. Studies have shown that experts and lay people perceive risks differently, particularly in areas of science and technology (Siegrist et al, 2007; van Heek et al., 2017). The news media, moreover, can play an indispensable role relaying knowledge from industry experts to the public. 
As AI could lead to less jobs or the creation of better jobs, potential benefits or disruptions to the future workplace arguably make AI a controversial topic. For this reason, this MRP seeks to explore public opinion on Reddit for the purpose of identifying the sentiments and beliefs that make up the discussions occurring on social media about AI and the future of work. Recognizing that experts and lay people may hold different views on the risk that AI poses to the workplace, this project's exploration includes an additional analysis of news articles shared on Reddit and the expert quotes contained within them. Taking an inductive approach, conventional content analysis of end-users' comments, of news articles, and of expert quotes within those news articles will reveal the themes and subthemes that make up the topics and sentiments that dominate the discourses on Reddit and in the news media in the context of AI becoming an occupational reality. A quantitative breakdown of the themes and subthemes and a qualitative review of the resultant observations will reveal which sentiments and beliefs form public opinion on Reddit as well as reveal how end-users, journalists, and experts similarly or dissimilarly view the impact of AI on the future of work. 


\section{Literature Review}

\section{AI and the Future of Work}

AI is more or less an all-encompassing abstraction of different ideas. AI can be understood as an overarching field that captures various methods found in computer science, or AI can be understood from associations made in popular culture. As the culmination of methods in computer science, AI falls into two categories: symbolic AI or computational intelligence. The former is based on the development of knowledge-based systems, or systems with the intelligence to give advice using information provided by human experts. The latter is based on the use of neural networks or evolutionary computing (Kalra et al., 2010). AI in the news media often appears alongside terms like machine learning (ML) and deep learning (DL) (McClelland, 2017). ML represents processes that allow machines to learn from data without the need for specific programming (Chah, 2019). DL is an extension of ML that consists of using artificial neural networks, or algorithms that replicate the human brain (McClelland, 2017). Other researchers have acknowledged the complexities of defining AI, as is the case with Simmons and Chappell (1988) who proposed, "The term [AI]...denotes behavior of a machine which, if a human behaves in the same way, is considered intelligent" (p. 14). Hereon AI, for the sake of conducting research, can be construed as an entity developed from complex intelligent systems that simulate human behaviour.

Industrial revolutions have occurred throughout history and have drastically changed, or even revised, the means of production. The first industrial revolution saw reduced dependence on human and animal labour from the proliferation of mechanization and steam power. Fordism and methodical assembly lines characterized the second, aided by the accessibility of electricity (Syam \& Sharma, 2018). The provenance of computers and job replacement due to digital 
technology marked the third, followed by developments in processing power and memory that facilitated the automation of delivering information (Syam \& Sharma, 2018). Evolving from this digital age, a fourth and ongoing industrial revolution materialized as a result of the sheer speed at which technologies developed and the wide range of different industries that had been impacted simultaneously. Of the technologies meshing areas of physicality, biology, and digitality, breakthroughs in AI and robotics—among others—-suggest that there will be a transformation of existing production, management, and governance systems (Schwab, 2016). AI and robotics emerging in a fourth industrial revolution signify a dismantling and reconfiguration of contemporary political and economic ecologies.

The integration of robots into the workplace poses broad economic implications resulting from occupational changes that alter how job-related tasks are completed. Machine workers eliminate the need for real people to engage in unpleasant, tedious, or dangerous work. The inorganic nature of machinery, moreover, protects robots from possessing sentience and precludes emotion from interfering with maximum productivity (Borenstein, 2009). Researchers Frey and Osborne (2017) argue that $47 \%$ of jobs in the US are at risk of being automated. Brookfield Institute at Ryerson University estimates this to be $42 \%$ for jobs in Canada (Lamb, 2016). Other countries present a less somber picture. Researchers estimate that approximately only $9 \%$ of Australian jobs may be automated. (Borland \& Coelli, 2017). According to calculations of countries in the OECD, an overall $14 \%$ of jobs are at risk of automation, but in the same report, Canada's numbers align much more closely with the global estimate. The OECD proposes that the percentage of jobs at risk for automation in Canada falls somewhere between 10-20\% (OECD, 2019). 
Premonitions such as these are not novel in the broader historical context as economists in the past have decried the consequences of technological unemployment (Keynes, 1930). It is important to point out, for this reason, that the economic extent of automation is dependent on several countervailing forces, like productivity (Acemoglu \& Restrepo, 2018). As highlighted earlier, several industrial revolutions have occurred as a result of technological progress. Remarkably, however, US historical patterns spanning the twentieth and twenty-first centuries show that, contrary to the grim outlooks currently proposed, when major industries in the past have automated, employment increased (Bessen, 2018). Some evidence exists illustrating a polarization away from middle skill occupations, but this polarization is unlikely to have any lasting consequences (Autor 2013; Autor, 2015). This is not to say that future jobs will not be impacted by AI. Autor (2015) points out that automation has traditionally sought to displace manual labour or error-prone cognitive tasks like data tabulation. Historically automation confined itself to the simplicities of routine tasks, but machines are more advanced today and continue to evolve. Generally speaking, making the assumption that other types of tasks are protected from automation, as they do not fall into the above categories, is to an extent fallacious. AIVA, for instance, is an AI software that composes compelling music compositions. Reading thousands of venerable music pieces and learning from them throughout the process, AIVA produces original and sentient symphonies (Barreau, 2018). Orchestral music composition is a skill that for humans arguably requires mastery of the field culminating years of experience and extensive studies. Grasping music production and possessing the ability to churn out songs on demand, AI-generated music highlights the potential impact that AI could have on professionals in creative industries. For example, mobile video game Pixelfield employed AIVA to create its main theme titled Battle Royale (Aiva, 2017). 
Despite the fact that there have been burgeoning advancements in AI, a machine with the capability to automate a certain job does not necessitate that it will gain the acceptance of the broader community. As social beings, humans may not support automation, or they may support the automation of one occupation more than the automation of another, such as automating bank tellers over food servers (Borenstein, 2009). For the same reason that humans are social beings, opinions are in flux and heterogeneity characterizes individuals' impressions of AI. Examining the beliefs of earlier researchers, Churcher (1991) proposed "AI automata" and "enhancing AI" as two views that emerged in academia and business. "AI automata" is AI in the exact sense of the word, receptive and reactive to cues in the environment. "Enhancing AI" is more or less representative of information sources or expert systems that support task completion and increase human efficiency at work. The categories are further broken down into "optimistic AI" and "pessimistic AI" as Churcher (1991) noticed that perspectives on AI and work-leisure were heterogeneous among earlier researchers. Inevitability and auspiciousness characterized the optimistic AI perspective; however, the same study observed that researchers who fell into this category did not discriminate between AI automata and enhancing AI. An example of the optimistic AI perspective would be someone indicating that the elimination of jobs creates more family time. The resultant message is one where AI automata and enhancing AI hold equal weight. That is, AI automates and benefits jobs simultaneously. Apparent distinctions, on the contrary, can be observed in the pessimistic AI perspective characterized by disruption and the concentration of power (Churcher, 1991). Fluctuating perspectives, to this end, between optimism and pessimism further corroborate the degree of controversy that surrounds the issue of $\mathrm{AI}$ and its implementation into the workplace. 


\section{Online Journalism and Social Media}

Perceptions toward AI may be mixed, but one thing remains almost certain about the future: the landscape of media is changing and journalism is moving online. As of 2018, one-infive adults in the US reported receiving their news from social media (Shearer, 2018). Digital readership is rising evident by the fact that the percentage of individuals receiving their news online is close to surpassing the percentage of individuals receiving their news from television. Visual broadcasting is the current most popular-but declining—news source (Shearer, 2018). New media like online journalism considers the technological capabilities afforded by digital platforms across the Internet. Whereas traditional journalism may have focused on the audience and the story, online journalism must consider interactions with the public, different media formats, and tools like hyperlinks to additional resources (Deuze, 2001). According to Kaplan and Haenlein (2010), social media consists of Internet-based applications that allow for the creation and distribution of content by and for end-users. Making audience engagement more accessible, social media has enabled end-users, including the news media, the potential to amass a large online following and consequently a large — or even larger — degree of influence. Usergenerated content (UGC) and social media are, accordingly, key components of new media because journalists must consider how readers will respond to news stories and share them online.

Reddit, a variant of social media, amalgamates the news media with the popular aspects that characterize the online social engagement features that appear across a variety of other social media platforms like Facebook, Twitter, and Instagram. Remarkably, as of 2019, 430 million end-users actively use Reddit every month, which further demonstrates the widening scope and prevalence of social media as a medium for news consumption (Murphy, 2019). Information 
received from news on social media has even been associated with positive impacts on knowledge acquisition. Previous research has observed that individuals who frequently used Twitter possessed more knowledge of current affairs (Boukes, 2019). In the same study, however, the opposite was true of individuals who frequently used Facebook. These results, according to Boukes (2019), suggest that external factors such as interfaces or affordances may affect social media's potential to bolster or hinder news consumption online.

The collaborative nature of social media provides individuals affordances that go beyond simple interaction. UGC, in addition to helping end-users maintain social relations and receive news, can be used to provide feedback, receive commentary, and communicate with others to accomplish collaborative tasks (Kaplan \& Haenlein, 2010). Social media platforms, for instance, like Wikipedia enable end-users the opportunity to create UGC for the purpose of facilitating the process of seeking information online. End-users have the ability to add new material or change existing material by removing or correcting inaccurate information. The philosophy behind collaborative projects, such as UGC on Wikipedia, is ultimately based on the idea that better outcomes result when people work together, not alone. Like wikis, social-bookmarking applications, such as Reddit, fall under the collaborative project umbrella. Social-bookmarking sites afford end-users the ability to rate and collectively promote links or news stories online (Kaplan \& Haenlein, 2010). Features like the upvote and downvote system on Reddit function to bolster or hinder the degree of exposure UGC gets.

Collaborative information sharing, particularly from the perspective of business, is similar to the efficient-market hypothesis, a finance theory that posits market prices fully reflect available information (Kaplan \& Haenlein, 2010; Fama, 1970). Amazon, for example, tested the implementation of dynamic pricing, and soon after, comments decrying the practice as unfair 
appeared on Wikipedia. The collaborative project against Amazon highlighted the need to recognize social media as a pivotal source of information for consumers (Kaplan \& Haenlein, 2010). UGC contributes to the dissemination of information, to this end, by facilitating news consumption, social engagement, and collaborative tasks. Together, these characteristics of UGC demonstrate social media as the future for receiving news and highlight the potential impact of social media on acquiring knowledge of current affairs or business ventures.

\section{Public Opinion and Science Deliberation}

Collective effort begins with the individual, and individual opinions are, in the exact sense of the word, opinions that are uniquely possessed and personal. No doubt others may feel or act differently in any given situation. In a broader context, such as one that is democratic, where freedom of speech fosters public deliberation, individual opinions can congregate and lead to a collective sentiment that represents the thoughts and opinions of the majority. This idea is conceptualized by the notion of public opinion. Shepard (1909) notes, "The term public opinion is used to describe both the sentiments and desires as well as the opinions proper which prevail among the individuals of a public" (p. 38). There are several other definitions of public opinion; however, a common thread exists throughout them. Most of the definitions are based on the aggregation principle. That is, public opinion is the culmination of separate and individual sentiments. Measuring and revealing public opinion has traditionally been accomplished through the use of surveys, polls, or elections (Herbts, 1993).

Perpetual exposure to the news media is a familiar encounter in Western cultures, and as a result, media effects arguably contribute to the topics that gain prominence and circulate in popular discussion among peoples living in Western societies. Previous research has shown this to be the case, with variation depending on the actor from whom the public receives their 
information. Statements made by news commentators have, in the past, observably paralleled substantial change in public opinion (Page et al., 1987). This media effect has also extended to stories that included experts or research studies, demonstrating that experts may have a similar impact on the public. For special interest groups, this media effect did not have a favourable outcome and appeared more dissuasive than persuasive. The rationale behind why experts may be more influential than other actors in the news media is possibly due to the perception that they are highly credible sources of information (Page et al., 1987). Researchers, more recently, have acknowledged the need to modernize the notion of public opinion due to the mainstream adoption of semantic polling. Anstead and O'Loughlin (2015), for instance, propose that public opinion represents not only the majority of individuals' views altogether, but also the majority of individuals' views in different publics. Technology evolves over time and as a consequence, ideas held by assemblages also evolve because new ideas are formed when individuals can, as a result of new mediums, contemplate popular views prevalent elsewhere (Anstead \& O'Loughlin, 2015). Social media platforms, for example, do not necessarily represent universal opinion, but social media platforms could very well represent a public, among many other publics, that influences conceptions of public opinion or from which measured public opinion emerges.

Venues for deliberation conceivably play a pivotal part in the formation of public opinion as communication invariably contributes to the way individuals receive and make sense of information. That said, temporal effects on the development of ideas are no doubt inevitable as influential societal actors such as academics, experts, and entrepreneurs, and the public are malleable, as is the rest of humanity, to the dynamic events subsumed within the chronological movement of progress. Three waves of science studies have occurred over the course of several decades (Collins \& Evans, 2002). The first wave espoused the belief that truth was, in essence, 
attainable only through rigorous training and traditional academic methods. Matters involving science and technology excluded the public, and experts spoke with authority and decisiveness. The second wave made alterations to this early understanding of science by redefining science as a social activity, where the legitimacy of experts could come under close scrutiny. While the second wave made scientific discussion more democratic by enabling social participation, Collins and Evans (2002) believe that in overcoming the Problem of Legitimacy that characterized the first wave, the second wave inadvertently created the Problem of Extension. In other words, "How far should participation in technical decision-making extend" (Collins \& Evans, 2002, p. 237).

Technical decision-making (the intersection of science, policy, and public interest) is a realm where experts and the public debate over issues of science and technology. The third wave proposed by Collins \& Evans (2002) reaffirms the importance of the second wave but argues for the addition of "a boundary around the body of 'technically-qualified-by-experience' contributors to technical decision-making" (p. 238). The three waves of science studies, in short, provide a glimpse of the roles that experts and ordinary people have had in shaping society's understanding of science and technology. While assessing the expertise of experts and lay people using social media is beyond the scope of this MRP, social media can be a forum for debate where lay people, and possibly experts, engage one another. When discussing current affairs regarding science and technology, end-users are, in essence, making technical decisions because they are deliberating over issues where their opinions may impact, and even influence, societal and political responses to emerging technologies.

Knowledge differences play an important role in deliberation, and empirical studies have attested to the existence of differences in risk perception between experts and lay people. 
Recognizing the influence of public attitudes on research and development, Siegrist et al. (2007) investigated public perception of nanotechnology. The results of their study revealed that experts and lay people viewed nanotechnology differently. Lay people perceived higher levels of risk and were warier of government intervention. Experts, on the other hand, had more trust in the government, and accordingly, its ability to reduce the health risks associated with nanotechnology. Differences between experts and lay people have also been observed in other areas of scientific inquiry. Investigating public perception of carbon dioxide utilization, van Heek et al. (2017) observed that experts and lay people had divergent opinions on carbon dioxide. Interview questions in their study included asking the subjects whether they believed that recycling excess carbon dioxide to manufacture plastics would be either sustainable or harmful. According to van Heek et al. (2017), lay people had a negative view of carbon dioxide, whereas experts had a view of carbon dioxide that was neutral and objective. A schism, to this end, has been observed between experts and lay people in relation to science and technology. Such observations highlight a potential dichotomy between two societal groups that both play an important a role in the construction and dissemination of knowledge about science or current affairs. From nanotechnology to carbon dioxide utilization, two similar, but different, technologies have demonstrated that lay people and experts have different responses to potential hazards. Similar findings may be observed in other areas where the introduction of new technology is seen as equally, if not more, inflammatory—such as AI and the risk of automation in the future of work. 


\section{Research Questions}

AI has the potential to disrupt and transform future ecologies, ultimately posing implications for the future of work (Schwab, 2016). The following research questions have been formulated based on a reading of the literature regarding AI, new media, public opinion, and technological deliberation. Viewing Reddit as an arena of public opinion and a platform that fuses social engagement with public deliberation and the news media, the below research questions were constructed as follows:

RQ1. How similar are the views in the news media, in expert opinion, and on Reddit regarding $\mathrm{AI}$ and the future of work?

RQ2a. What sentiments guide public discourse online about the changing nature of work?

RQ2b. What specific sentiments and views are the most prominent on Reddit regarding the changing nature of work and $\mathrm{AI}$ ? 


\section{Methodology}

\section{Background on Reddit}

Social media bolsters the dissemination of news online by allowing end-users to take control of some of the more traditional gatekeeping practices that have long influenced individuals' exposure to the news media. Access to social media provides end-users the option to play an active role questioning the veracity of news stories by viewing commentary in real time as well as the option to play an active role deciding which news stories are worth sharing with others. Social media, ultimately, makes the dissemination of news much less unidirectional. The main purpose of social media, however, is not news dissemination. Facebook, Twitter, and Instagram are social media platforms where individuals can follow and stay in touch with friends, family, and, more recently, influencers. Reddit differentiates itself as a social media platform by, more or less, exclusively focusing on the dissemination of news, primarily limiting social interaction to public deliberation over the content of shared links and articles. Researchers have, in light of this, even referred to Reddit as a social news site (Weninger, 2014).

Reddit affords end-users the option to create and participate in virtual communities called subreddits. In these subreddits is where social engagement and sharing news occur. These individual online communities are created to represent topics of interest that end-users wish to discuss, but content can become so popular that it goes viral and, consequently, reaches audiences beyond those actively involved in the subreddit from which it came. The process of sharing information in subreddits is relatively simple; end-users interact with one another by posting forum-like threads known as submissions, and each submission includes a section for comments where end-users respond to the post itself or respond the comments that had been left by other end-users. Threads typically consist of links to news articles while the comments 
section consists of end-users deliberating over what they had read. Reddit also gives end-users the option to upvote or downvote content. Threads and comments, accordingly, can either increase or decrease in visibility depending on the number of upvotes or downvotes they receive from end-users.

\section{Methods and Data Extraction}

Incorporating qualitative and quantitative techniques, the research followed a mixedmethods design to identify, extract, and analyze data from Reddit. Several types of qualitative content analysis are available to organize and examine phenomena in communication texts. Conventional content analysis is a well-suited inductive approach to investigate data when there is a paucity of developed theories in the literature that could be used as means to perform deduction (Hsieh \& Shannon, 2005). This inductive approach for content analysis consists of observing the chosen communication texts for similarities and dissimilarities and, subsequently, grouping the observations into categories or themes that emerge as a result of investigating and interpreting the manifest and latent content (Graneheim et al., 2017). Categories and themes are often understood in the literature to be homogenous, and, consequently, both terms are used interchangeably. Subtle differences, however, are perceptible. Themes are distant from the text, as compared to categories, and require a high degree of hermeneutics. Descriptions of themes are abstract and reflect an above minimum interpretation of the studied object. Categories, on the other hand, are closer to the text and represent phenomenological descriptions that require much less interpretation. Aiming for objectivity, categories are more concrete and attempt to emulate texts at face value (Graneheim et al., 2017). To create new themes, an inductive framework was used to categorize communication texts from Reddit and the news media in order to identify observable similarities and dissimilarities. Developed themes from the collected discourses 
would reveal popular topics emerging from relevant discussions about AI and the future of work. As the research questions were also interested in examining sentiments, the communication texts were additionally investigated to reveal attitudes. The sentiments in this study are indicative of developed subthemes that resulted from discernable attitudes in the collected discourses. Categorized using the same inductive approach used for the themes, the subthemes reflect prominent sentiment patterns that emerged after sorting the data into groups based on similarities and dissimilarities. The research, to this end, conducted inductive content analysis by coding manifest and latent content into themes and subthemes from discourses on Reddit and in the news media.

Using a similar approach suggested by Bengtsson (2016), the methodological framework followed a step-by-step process in order to perform inductive content analysis. The project began by identifying the aim of the study which was to gauge public opinion on Reddit regarding AI and the future of work and observe similarities and dissimilarities between the sentiments of lay people in comparison or in contrast to the sentiments of experts and journalists in the news media demonstrating a degree of legitimacy or informed knowledge in the field of AI. Previous research has found that the informational use of social networking sites to obtain news was positively associated with online and offline political participation (Gil de Zúñiga et al. 2012). Reddit promotes news dissemination, and end-users actively engage one another online to debate over current affairs. In light of social media facilitating public deliberation, news articles contained in Reddit submissions and the comments within those submissions were chosen as units of analysis for the research. Because news articles also contained quotes from experts these, too, were selected as a unit of analysis. 
In order to gather a sample that would allow the research to accurately identify themes in the communication texts, the top submissions that pertained to AI and the future of work were chosen. These submissions reflected content that garnered the most support from end-users because they had collectively upvoted that content to increase its visibility. Data from the top submissions were used to perform the inductive coding portion of the research. Strategic keywords were used to find the pertinent Reddit threads by searching for the words "artificial intelligence" and "jobs" contained within the title of submissions across the social news site. Once the keyword search had revealed the most popular threads, the top 3 were selected in addition to the comments, news articles, and expert quotes within those news articles. Because submissions on Reddit can contain thousands of comments as well as sub-comments, the research design chose only to look at the top 12 "best" comments in each of the top 3 submissions. Comments that were not relevant to the research were skipped. The discourses were subsequently parsed and decontextualized into meaning units which were then recontextualized by rereading the meaning units next to the original texts in order to determine whether or not any remaining uncoded texts would contribute to answering the research questions.

Categories were formed by combining meaning units into manifest categories based on the observable similarities and dissimilarities of the data. The entire process consisted of interpreting the manifest content and gradually incorporating a higher degree of hermeneutic interpretation in order to develop broad themes that were based on the manifest categories that had emerged as a result of this process. Morse (2018) proposed that the creation of themes is a process of seeing the data implicitly and asking what the content is about; following this concept, the manifest categories were grouped based on their implicit presentation, leading to the creation of themes and subthemes. The themes that were identified as a result of inductive content 
analysis were inequality, economy, and economy/inequality. The subthemes that were identified were hope, dread, and neutral/mixed.

Once the themes and subthemes had been created and turned into codes, the research coded data from the top 8 Reddit submissions related to AI and the future of work. The design additionally expanded data collection from the 12 best comments to the best 15 comments. Because the second top submission on Reddit had the same media article as the first top submission, the article and the expert quotes within this article were only coded once. An additional submission was added to reconcile the loss of the data. The associated comments in this additional submission were excluded because comments in the second top submission had been coded. Overall, 125 discourses were gathered. Of that total, 13 discourses came from expert quotes, 7 from journalists, and 105 from end-users on Reddit. Descriptive statistics were used to expand on the identification of the themes and subthemes. Recording the frequency of the themes and subthemes enabled the quantification of the qualitive data, which added another layer of comparison between the units of analysis. What follows in the analysis section introduces the themes and subthemes further and provides a quantitative breakdown of the themes and subthemes as they appeared in online discourses on Reddit, in the news media, and in expert quotes pertaining to $\mathrm{AI}$ and the future of work. 


\begin{abstract}
Analysis
An analysis of the data gathered from Reddit submissions and the associated news articles and expert quotes revealed several prevailing themes and main ideas regarding AI and the future work. Economy and inequality dominated the discourses as the primary themes that emerged from the topics or concerns that Reddit users, journalists, or experts quoted in the media discussed. Subthemes of sentiment emerged in parallel that contextualized the opinions or thoughts expressed by the units of analysis. AI, for the most part, was not seen in the data as a neutral technology, and consequently, there were no lack of individuals online sharing their thoughts and opinions about the implications or non-implications of advanced AI entering the workplace. For a more extensive overview or in-depth look at the themes and subthemes, a codebook of the inductive findings can be found in the appendices of this MRP; however, succinct breakdowns of the major themes and subthemes, as well as graphed depictions of the data, are provided throughout the subsequent pages of the analysis chapter.
\end{abstract}

\title{
Quantitative Overview of Themes
}

The theme economy represents a perspective where individuals made associations between $\mathrm{AI}$ and varied facets of the economy, and to no surprise, the economy theme was the most frequent theme that appeared in the discourses. In the literature, many academics expressed that AI would be a technology that would have economic implications (Frey \& Osborne 2017). Appearing 57 times overall, the theme economy represented $45.6 \%$ of the topics subsumed within the pulled data. The theme economy/inequality held the title of the second most frequent theme overall. As a category, the theme economy/inequality is a hybrid theme that, as its name implies, conjoins aspects of both inequality and the economy. This theme represents discourses 
that emerged from the data where individuals, regardless of the data source, associated AI with broad implications that extended throughout society and the economy.

Table 1: Frequency Distribution of Themes for Overall Data

\begin{tabular}{|c|c|c|}
\hline Theme & Frequency & Percent \\
\hline Economy & 57 & $45.6 \%$ \\
\hline Economy/Inequality & 46 & $36.8 \%$ \\
\hline Inequality & 22 & $17.6 \%$ \\
\hline Total & 125 & $100 \%$ \\
\hline
\end{tabular}

Note. The economy theme represents over half of the discourses.

The rationale for labelling and, consequently, creating the hybrid theme economy/inequality was ultimately due to the prevalence of both themes inequality and economy being manifest in the same discourses. The theme economy/inequality, for this reason, was developed to capture thematic diversity, and discourses that matched the criteria for both themes were categorized into this separate hybrid theme that represented a unique variant of the communication texts. With a frequency of 46 , the theme economy/inequality made up $36.8 \%$ of the overall data. Inequality, the last of the major themes, was less frequent than both the theme economy and the theme economy/inequality. Discourses labelled inequality represented texts that, in essence, highlighted conflicts or societal divides that would be either exacerbated or equalized from the introduction of AI into the workplace. The theme inequality made up only $17.6 \%$ of the overall associations or topics that were subsumed within all of the end-user, journalist, and expert discourses collected.

While the frequency distribution of the major themes showed that the economy theme appeared the most frequently, a more detailed breakdown of the major themes illustrated that the frequency of the themes differed depending on the source of the data. Whether the data came 
from expert quotes or news articles, the frequencies of the themes within those units of analysis were, with the exception of end-user's comments, different than the distribution of the major themes in the data as a whole. For experts, specifically, economy and economy/inequality were the primary themes that appeared in the discourses. The frequency of the theme economy/inequality was the same in expert discourses and journalist discourses. The theme inequality for experts and journalists appeared the least frequently, similar to the overall data, but with a frequency of 3, inequality in expert quotes appeared almost as often as did the first and second most frequent themes, economy and economy/inequality.

Figure 1: Frequency of Themes in Journalists and Experts

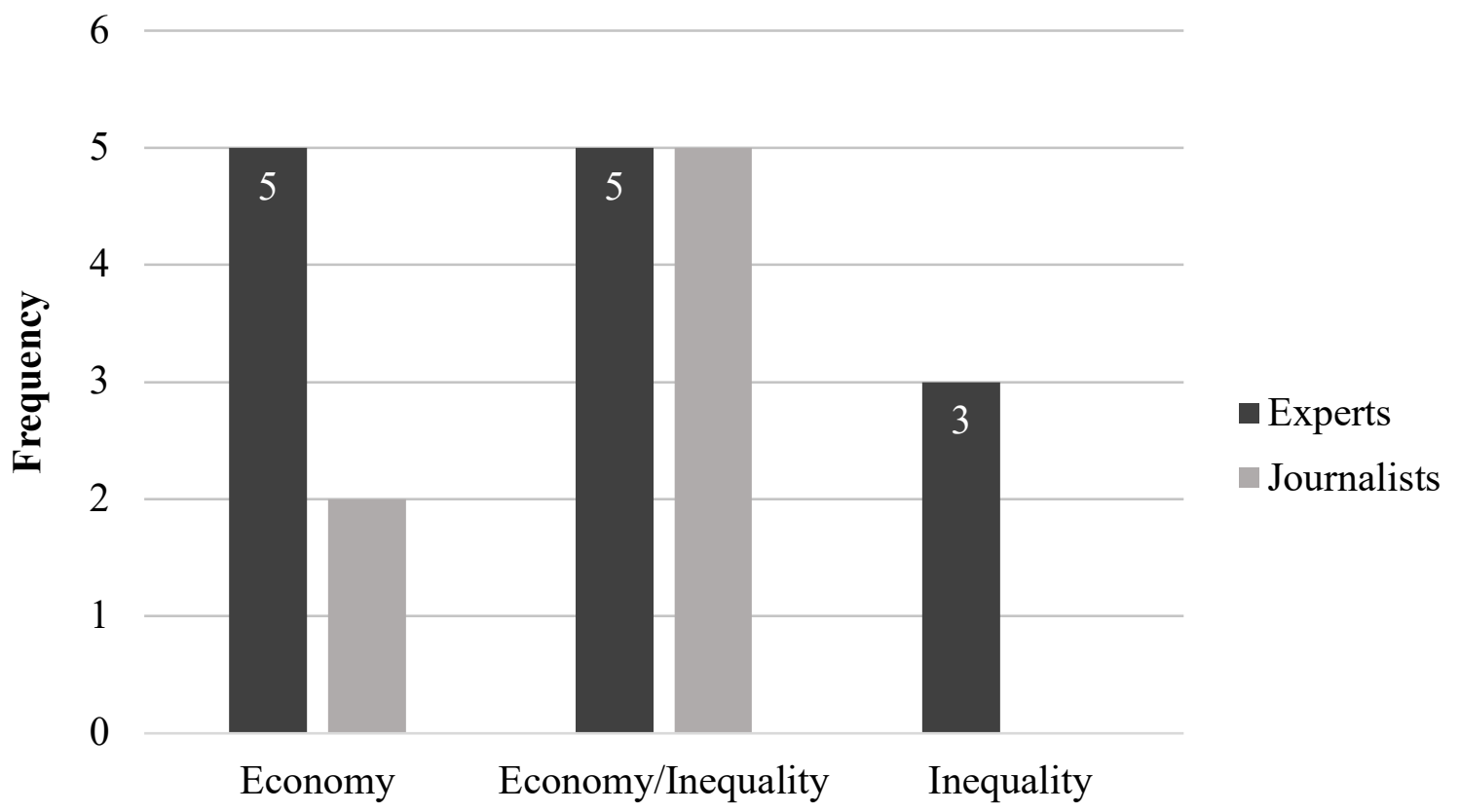

Theme

Note. The economy/inequality theme is the most frequent altogether.

In the news media, the most popular theme that emerged in the journalist discourses was the theme economy/inequality, with the theme economy following shortly thereafter. The theme inequality alone was not observed in the media. The frequency of the theme economy/inequality was 5 while the theme economy appeared only twice. The frequency of the themes in the 
discourses of end-users on Reddit was similar to the frequency distribution of the major themes overall. The theme economy was the most frequent theme to appear for end-users on Reddit. Of the total 105 Reddit comments investigated, the theme economy was observed in 50 of them. The theme economy/inequality was found in another 36 comments out of the 105 comments. The theme inequality was the dominate theme in the remaining 19 end-user discourses.

Some distinct patterns emerged during an observation of the various frequencies, overall and by theme, in the discourses of Reddit users, journalists, and experts. Aforementioned in the previous paragraph, the theme economy is the overall most frequent theme when all of the units of analysis are aggregated into a frequency distribution. A similar finding is also observed when looking at the frequency distribution of the themes that appeared in the discourses of end-users. When excluding this unit of analysis (end-users), an examination of the other two units of analysis amalgamated (expert quotes and news articles) revealed the economy/inequality theme as the most frequent theme to emerge.

\section{Qualitative Overview of Themes Analysis}

In order to contextualize the quantitative analysis of the themes that were presented in the previous frequency distributions which reflected the amount of appearances of the major themes overall and by unit of analysis, a qualitative examination was done to investigate the main ideas and beliefs that appeared most frequently alongside the themes in the communication texts. This analysis enriched the data by contextualizing the observed similarities and dissimilarities in the discourses and contributed to the findings for RQ1. As end-users demonstrated an affinity for the theme economy, a deeper examination of the apposite discourses was conducted to elucidate the main ideas behind the prevalence of this theme. The examination illustrated that most end-users focused on the current economic infrastructure as the biggest mediator between the good, the 
bad, or the bland possibilities of the future that would follow the introduction of increasingly advanced AI. End-user E1 (2018) posted the following:

Just like agricultural workers massively moved into industrial production in the late 1800 s, people will have to retrain themselves for whatever comes next. Many people seem to think that nothing WILL be next, and envision a world where A.I is the only necessary input for production, with humans playing little, or, ultimately, no part. While no one probably knows for sure, there is one thing we DO know : the limitless nature of human desire for a better life.

The author of the above quote uses history as an example to form a probable estimate of what the future of AI and employment could look like. The depiction of history provided is one where employment has remained resilient in the face of mechanization. Rather than succumbing to mass unemployment, individuals had adapted and acquired new skills for work in new industries, and a better life, henceforth, ensued. Possessing a slightly different perspective, end-user E2 (2016) made the following comment:

I'm going into the tourism industry and I'm having a hard time processing how the advancement of AI will impact my job security. A bi $[\mathrm{g}]$ part of tourism is making experiences "authentic", tailor-made for each individual and "emotional" in a sense. I can only imagine a person being able to supply this kind of service in the lifetime that I'll be alive. But on the other hand, seeing those self-service machines at mcdonalds (which I use every single time because of how simple and effective they are), makes me think that maybe the service that I will be providing isn't that hard to pull off by a program anyways.

Unlike the first end-user, the second end-user looked to the impacts of mechanization in the present. The writer, however uncertain about the future, identified the limitations of AI to be an inability to simulate ingenuity, dexterity, and sentience. These traits happen to be the uniquely human attributes that engender human creativity and make human innovation possible.

Additionally, as the writer puts it, these distinctly human attributes preclude AI from automating entire industries, particularly those industries that rely heavily on these very human faculties.

Both comments reflect the main ideas that were apparent in the economy theme from end-users: 
the economic infrastructure and its ability to cope with technological unemployment according to historical precedent.

On the other hand, as the data has shown, the theme economy/inequality dominated the discourses from journalists and experts. An examination of the overall discourses from journalists revealed that the main ideas and perspectives of the theme economy/inequality in the news media focused on accountability and the need for government and/or the need for policy intervention. Forbes journalist Koetsier (2016) argued that the public sector is ill-equipped to deal with the probabilistic disruption that will result from mass automation:

The challenge of new technology and new efficiencies, of course, is that our economy is built on people getting jobs, getting paid, paying taxes, and earning a living. Oxford researchers have estimated that half of all jobs are vulnerable to disruption from artificial intelligence, robots, and automation. In that scenario, massive social change and upheaval would follow, as half of the workforce loses its jobs, and some new kind of social contract -- even, perhaps a guaranteed minimum income -- could be a necessity. (para. 5)

Because some employment predictions have dictated that society and the economy would undoubtedly face an onslaught of technological unemployment, journalists such as Koetsier (2016) have made a point of simply stating that "Governments [are] not prepared." The idea, simply put, is that the consequences of AI will be widespread and will not just affect occupational realities, but also affect livelihoods and the way people will need to accumulate wages to afford the basic necessities needed to prosper in a system that is reliant on an employed populace.

Others have noted that while AI may not necessarily be job displacing, the introduction of AI into the workplace still warrants coordination between governments and industries in order to ensure an equitable and fair present and future society. Journalist Rosenbaum (2018), expanding on ideas from a quoted expert, wrote of the importance of including a diverse range of 
employees, cultures, languages, and practices in the development and environment of AI. All of which are important contributors to ensuring the impartiality of robot interaction with humans. According to Rosenbaum (2018), "Companies need to have a diverse human pool with different academic backgrounds to come together and decide what a platform is really about and how a company wants it to serve users" (para. 26). AI is no different than other technologies in that AI functions as a result of its programming. Engineers have the core responsibility to make sure that the programs they develop for AI are free of biases and prejudices.

The scope of AI development, however, extends beyond innate programming alone because experiential learning also affects the way AI behaves. For this reason, diverse exposure to different cultures and cultural practices is an important, if not fundamentally essential, component of ensuring that AI will learn and contribute to the workplace in an equitable and just manner. Impartiality is even more important when AI will take over occupational roles where their workloads indirectly or directly impact consumers and everyday people. Governments and policy workers, therefore, must work alongside engineers and corporations to ensure the neutrality of AI, such as by advocating the need for more jobs from the global market and ensuring that a diverse range of employees work with and alongside AI during research and development.

The qualitative analysis of the discourses from experts unearthed that the main ideas of the theme economy/inequality in expert quotes centred on AI as a technology with invariant promise. The future impact of AI, that is, is not a result of AI itself, but the way society and the economy react and make use of what AI could do. Industry professional Mohit Joshi (as cited in McKendrick, 2018) highlights the newfound reality AI may create: 
"So far, the arc of AI leans toward empowerment and giving humans the tools necessary to automate redundant tasks, detect and analyze hidden patterns in data and generally make possible revolutionary insights that will make our lives better" (para. 4).

As Joshi points out, AI has the potential to automate routine jobs that take away from other pursuits that are better oriented to the human condition. Liberated from tasks that are perhaps arduous, mundane, or even dangerous, humanity can spend more time focusing on ways to improve the world and on ways to create a more just and equal society where everyone benefits from having to work less. A new era of better work means that revolutionary insights like a universal basic income could become a reality. The future of AI will look more like a utopia as humans will be free from the contemporary 9-to-5 constraints that have generally characterized contemporary office and manufacturing work. Political thinker Matt Bruenig (as cited in Tarnoff, 2017) possessed a gloomier outlook of AI:

"The problem with robots is not the manufacturing and application of them - that's actually good for productivity. The problem is that they are owned by the wealthy, which means that the income that flows to the robots go out to a small slice of wealthy people" (para. 14)

AI is a promising area of technology that can benefit industry and the lives of everyday individuals. Increased productivity could mean more goods at a cheaper price for more people; however, benefits like improved production are not without the potential for serious ramifications. The automation of manufacturing jobs could very well lead to widespread job displacement as monetary gains from mechanization incentivize business owners and corporations to forego providing wages to workers. The salaries would be redistributed back to the owners of the AI, and average workers would be left impoverished. Such ideas demonstrate how AI can be a tool from which society benefits or a tool from which society harms itself. 


\section{Quantitative Overview of Subthemes}

Subthemes emerged from the data in addition to the major themes. Subthemes reflecting sentiment were apparent as individuals in the study were expressive in their discourse regardless of where they stood as a unit of analysis. The three identified sentiments were hope, dread, and neutral/mixed. Dread reigned over the discourses altogether for end-users, experts, and journalists. The aggregation of the sentiments showed that dread made 62 appearances, followed by hope which made 42 appearances and neutral/mixed which made 21 appearances. The subthemes, as mentioned previously, reflect the attitudes that appeared in the communication texts and were categorized as a result of inductive content analysis. Dread, hope, and neutral/mixed, more specifically, were identified by observing the discourses and coding the texts based on the manifestation of positive, negative, or ambiguous ideas that reflected the writers' feelings toward the future of AI and work.

Table 2: Frequency Distribution of Subthemes for Overall Data

\begin{tabular}{|c|c|c|}
\hline Theme & Frequency & Mean \\
\hline Hope & 42 & $33.6 \%$ \\
\hline Neutral/Mixed & 21 & $16.8 \%$ \\
\hline Dread & 62 & $49.6 \%$ \\
\hline Total & 125 & $100 \%$ \\
\hline
\end{tabular}

Note. The subtheme dread represented approximately half of the discourses.

The subtheme dread, as noted, represented one of several affective expressions that emerged from an investigation of the discourses from the units of analysis. Craft (1992) believed dread expressed, "the fear of the unknown, the apprehension of a future heavy with the possibility of danger" (p. 521). Conceptualizing the sentiment of dread similarly, the research in this MRP viewed dread as an apprehension about the future. This form of apprehension was 
observed throughout the collected discourses. Dread manifested much of itself in texts that contained foreboding ideas about AI and the future of work. The future of AI, however, remains unknown, including its potentially disastrous effect on employment or the distribution of resources. While negative attitudes about AI's trajectory could have been categorized as pessimism, the future of AI remains largely speculative — even among experts. The sentiment dread, for this reason, was chosen and, subsequently, made into a subtheme because it reflected an attitude that considered the negative, unknown, and hazardous possibilities of the future. Of the subthemes, dread appeared the most frequently, and from the perspective of popular culture, this abundance of dread in the discourses is not unforeseen. Box-office hits like The Terminator have long created the impression that AI, especially when left to match or surpass human intelligence, would, at one point in time or another, lead to the assured destruction of humanity because sentient robots would seek retribution for being an exploited technology ${ }^{1}$.

The second most frequent sentiment that was revealed from coding the data was hope. Like dread, hope is a future-oriented emotion (Bruininks \& Malle, 2005). Rather than dreading what may happen, hope looks forward to what could happen. Hope is unafraid of a future full of possibilities. Whether things change slightly or things change in a way that emerging technologies drastically improve everyday lives, hope is confident that the future will be socially or economically better off than the present. Bruininks and Malle (2005) elaborate on hope further:

Often the individual perceives little personal control over the situation. The individual may see a positive outcome as somewhat unlikely and yet still expect it to occur. Thus, being hopeful enables people to maintain an approach-related state despite their present inability to reach the desired outcome. (p. 338)

\footnotetext{
${ }^{1}$ See Dockterman (2019), for release date and Terminator franchise storyline.
} 
In the discourses, individuals that demonstrated feelings of hope possessed the opinion that AI would not turn into some malevolent entity that would ultimately lead to the widespread destitution of working people regardless of their occupation. Whether or not jobs would be automated, hopeful individuals foresaw a promising future where humans still had an important role to play. Although, for a small minority of radical end-users, AI was, what they anticipated to be, a means of creating the necessary conditions to incite the communist revolution for which they had been waiting. While discourses with positive attitudes could have been categorized as optimism, views such as the one provided most recently show that the sentiment hope more accurately reflects opinions on the issue of AI. The small minority of end-users referenced above, for example, may believe that AI will invariantly lead to more conflict, but theyexhibiting a sense of hope - also believe that AI will eventually lead to less disparity between social classes.

The last and least frequent sentiment subtheme was neutral/mixed. The existence of this subtheme emerged largely out of the ambiguity that characterized discourses that were neither partial nor definite. Individuals subsumed within this subtheme were markedly indifferent or demonstrated impartial reservations about AI. Previous research has looked at impartiality on social media. Zafar et al. (2016), in studying social media texts, foregrounded an intuitive notion of affiliation-discernibility in order to determine the partiality of discourses on social media. Affiliation-discernibility, in brief, dictates that the degree of challenge an observer has confidently discerning the affiliation of a writer based on their text determines the degree of that writer's message's impartiality. While the methodology of this MRP did not follow the affiliation-discernibility method, the identification of the subtheme neutral/mixed was the result of a similar process. Stances on the issue of AI and work could not be easily discerned in some 
of the texts pulled from social media or the news media and, consequently, these texts could not be categorized as either dread or hope. The neutral/mixed subtheme reflected discourses that either weighed the negative and positives impacts of AI equally or simply depicted messages that were wanting the attitude of the writer.

Table 3: Frequency Distribution of Subthemes by Unit of Analysis

\begin{tabular}{lcccccc}
\hline Unit of Analysis & \multicolumn{6}{c}{ Subtheme } \\
\hline & \multicolumn{2}{c}{ Hope } & \multicolumn{2}{c}{ Neutral/Mixed } & \multicolumn{2}{c}{ Dread } \\
\cline { 2 - 7 } & Frequency & Mean & Frequency & Mean & Frequency & Mean \\
Experts & 5 & $38.4 \%$ & 3 & $23 \%$ & 5 & $38.4 \%$ \\
\hline Journalists & 3 & $42.8 \%$ & 1 & $14.2 \%$ & 3 & $42.8 \%$ \\
\hline End-users & 34 & $32.3 \%$ & 17 & $16.1 \%$ & 54 & $51.4 \%$ \\
\hline
\end{tabular}

Note. Slightly over half of end-users exhibited a sense of dread.

Further quantitative analysis of the data was conducted to show the distribution of the sentiments by source. The most frequent sentiments that appeared in expert quotes were dread and hope. Both sentiments made 5 appearances respectively. The subtheme neutral/mixed was observed the least, with only 3 appearances. The frequency of the sentiments in discourses from journalists was strikingly similar to the frequency of the sentiments in the expert quotes. Hope and dread were, again, the most frequent sentiments that could be observed after a tabulation of the data, and again, like the subthemes for experts, the subtheme neutral/mixed was the least frequent sentiment to make an appearance, only showing up once for journalists.

With the exception of the subtheme neutral/mixed being slightly less frequent in news articles, there were conspicuous parallels between the frequencies of the sentiments in the content from both experts and journalists. The same could not be said for end-users providing commentary on Reddit. Dread was significantly more frequent for end-users than the subthemes hope and neutral/mixed. The frequency of dread, in fact, even outnumbered the frequency of the 
other two subthemes combined. On average, a little over half of end-users dreaded AI's integration into the workplace. Hope was observed in $32.3 \%$ of end-users, and in the remaining $16.1 \%$ of end-users, neutral or mixed feelings were expressed. Similar to the frequencies of the subthemes in the news media and in expert quotes, the subtheme neutral/mixed was the least frequent subtheme in end-users.

Table 4: Frequency Distribution of Themes and Subthemes

\begin{tabular}{|c|c|c|c|}
\hline \multirow[b]{2}{*}{ Unit of Analysis } & \multirow[b]{2}{*}{ Theme } & \multicolumn{2}{|c|}{ Subtheme } \\
\hline & & Hope & Dread \\
\hline \multirow[t]{3}{*}{ Experts } & Economy & 2 & 1 \\
\hline & Economy/Inequality & 3 & 2 \\
\hline & Total & 5 & 3 \\
\hline \multirow[t]{3}{*}{ Journalists } & Economy & 1 & 0 \\
\hline & Economy/Inequality & 2 & 3 \\
\hline & Total & 3 & 3 \\
\hline \multirow[t]{3}{*}{ End-users } & Economy & 19 & 20 \\
\hline & Economy/Inequality & 9 & 21 \\
\hline & Total & 28 & 41 \\
\hline
\end{tabular}

Note. The prevalence of hope in experts and journalists highlights the salience of the subtheme dread in end-users. Data reflecting the theme inequality and the subtheme neutral/mixed were omitted for clarity.

As the theme economy and the subtheme dread were the most frequent theme and subtheme in the discourses altogether, an examination of discourses containing both was performed. The subsequent examination revealed that, while the subtheme dread may have been the most frequent subtheme overall, individually the subtheme hope appeared almost as often as the subtheme dread in discourses containing the theme economy. This observation was particularly evident in end-users, although with 20 compared to 19 appearances, the subtheme dread had the advantage of being slightly more frequent than the subtheme hope. 
A deeper investigation into the data revealed that the subtheme dread, while perhaps not so distinct in end-users discussing the economy, was very distinct in end-users' comments that exhibited the theme economy/inequality. For example, $58.3 \%$ of end-users referencing both inequality and the economy in their discourses felt a sense of dread as compared with the $25 \%$ of end-users that were hopeful and whose discourses fell into the same economy/inequality category. The sentiment hope was, more or less, the dominate subtheme in expert quotes, particularly when discourses with the theme economy and discourses with the theme economy/inequality were amalgamated. In journalist discourses, the subthemes hope and dread appeared with equal frequency when discourses with the theme economy and discourses with the theme economy/inequality were similarly added together. When data from expert discourses and journalist discourses were combined, the resultant aggregation demonstrated that hope was the predominant sentiment for experts and journalists.

\section{Qualitative Overview of Subthemes Analysis}

Similar to the qualitative review of the themes, a qualitative review of the data on the subthemes was conducted in order to contextualize the observations made as a result of putting the sentiment data into frequency distributions. The qualitative review of the subthemes advanced the findings further by permitting a more thorough examination of the quantitative observations that provided glimpses of the sentiments and beliefs that guided discourses on Reddit regarding AI and the future of work. Augmenting insight into the discourses provided an element of depth to the data that was needed for the subjective component of $\mathbf{R Q 2} \mathbf{b}$. With added context assumptions could be made that helped clarify why some individuals on Reddit or in the news media dreaded the future of AI while others embraced the it with open arms. As RQ2b 
focused solely on the sentiments of end-users, only the data on communication texts from Reddit were reviewed.

In discourses characterized by aspects of the economy, sentiments were split among endusers somewhat uniformly. When the economy was the primary and only concern, roughly half of end-users felt a sense of hope or dread in regard to the possibility that AI would become increasingly integral to future workplaces. A review of the economy themed discourses revealed that the individuals that felt hopeful about $\mathrm{AI}$ and the future of work felt that way because they perceived the negative outcomes of mass automation to be alarmist and out of sync with the current economic realities or previous histories that were marked by periods of mechanization, such as the information age. Take, for instance, this quote from end-user E3 (2017) about the economy and the future of work:

The vast, vast majority of the $1 \%$ 's wealth is stock market valuations. Look at the list of America's Top 20 Richest People - every single one of them is there because of their stock holdings and almost nothing else. All those valuations, utterly $100 \%$ depend on a sound financial system - with wages earners able to buy products to give business revenues \& pay back all their consumer/student/mortgage/business/car debts. The one thing you can $100 \%$ guarantee is that if we have a world where most people are unemployed or poor - our financial infrastructure that supports stocks, pensions and high property prices collapses too.

The end-user provided an expository description of the current economy and the fallout that would subsequently occur if mass automation were to leave most of society without paid work. The current economic infrastructure is not only incompatible with the possibility of mass automation but also a buffer against widespread unemployment. Individuals would work together to preclude technological unemployment because the consequences would have ramifications at all levels of society. End-users that possessed a sense of dread about the economy, on the other hand, felt that way because they saw AI as a technology that has advanced, will continue to advance, and will ultimately become sophisticated enough to displace workers in occupations 
that were once thought to be protected from mechanization. End-user E4 (2016) felt a sense of dread about the future economy and went as far as using the trucking industry as an example to illustrate the potential impact of automation:

Let's break it down here, how many positions are going to be wiped out by AI once they become mainstream? Off the $t[o p]$ of my head, trucking is gone, most services are gone (fast food, travel agencies, restaurants), anything that does not require a strictly human touch is going to be mostly outmoded. Sure there may be some platforms that use this to their advantage, but it's up to the consumer to decide if the increased price for these "humanized" businesses is a respectable investment. Your option as an employee is to get educated now and cement yourself in a position that cannot be automated. Good luck, it was claimed the trucking industry is immune. Not going to be the case in 10 years boys and girls.

AI, as presented above, will inevitably displace jobs and only those occupations which rely on distinctly human faculties will be spared from displacement. Interestingly, this end-user mentioned human social relations as a potential mitigator for unemployment as society could collectively band together to boycott businesses that hire AI in favour of human workers. Giving an unfavourable ultimatum, this end-user proposed that employees can protect themselves by acquiring new skills that cannot be automated. New skills may not suffice, however, because according to this end-user the likelihood that AI will eventually automate most work-related tasks is high. 


\section{Findings and Discussion}

Having completed a qualitative and quantitative review of the data achieved through inductive content analysis, research conducted by this MRP observed and subsequently recorded notable patterns that emerged as a result of analyzing discourses on Reddit and in the news media. The quantitative review was conducted to identify and quantify the themes and subthemes that prevailed among end-users, journalists, and experts. The qualitative review was conducted to contextualize the results of the quantitative findings. Added context allowed for a richer description of the similarities and dissimilarities that were identified throughout the research process. The quantitative measurement and qualitative examination of the major themes answered RQ1 by revealing the thematic and conceptual similarities and dissimilarities between the units of analysis. The quantitative measurement and qualitative examination of the sentiment subthemes answered both RQ2a and RQ2b by identifying the prevailing sentiments and predominant views that culminated in public opinion on Reddit.

RQ1. How similar are the views in the news media, in expert opinion, and on Reddit regarding AI and the future of work?

Media: Economy/inequality was the most frequent theme to appear in the news media as the majority of the stories written by journalists predominantly discussed the economic and social ramifications of AI's current and future occupational role. The theme inequality did not appear on its own in the news media. Journalist discourses with the theme economy/inequality centred around ideas of accountability, particularly foregrounding issues like the need for government oversight of the research and development of AI technology and the need for 
government and policy intervention to mitigate the potential ramifications that could result from $\mathrm{AI}$ in the workplace.

Experts: With the same number of appearances, the themes economy and economy/inequality were the most frequent in expert quotes. Inequality appeared almost as often as did economy and economy/inequality respectively. Whether the pertinent discourses highlighted future employment or future employment and equity, expert quotes foregrounded AI's potential to change the world. This was especially the case for the theme economy/inequality.

End-users: Similar to the overall frequency of the themes, economy was the most frequent theme in end-users. The theme economy/inequality was the second most frequent, and inequality was the least frequent. A review of the theme economy in end-user discourses revealed that perceptions of $\mathrm{AI}$ in the workplace were largely dependent on viewing the current economic infrastructure as a potential mediator that would either act as a buffer or driver of unemployment.

\section{Discussion}

Aforementioned in the analysis section, a distinct pattern emerged from a comparison of the frequency distributions between journalists and experts. The themes economy and economy/inequality share the same frequency in expert quotes; however, the theme economy/inequality becomes the most frequent theme when the discourses from experts and from journalists are aggregated. Such an observation could be construed as evidence of the different ways journalists, experts, and lay people interpret and make sense of new information. The high prevalence of the theme economy/inequality, for instance, in journalist and expert discourses may suggest that journalists and experts, compared to end-users, are more perceptive 
of the implications of advanced AI in the workplace. Discourses that delineate both the social ramifications and economic ramifications of $\mathrm{AI}$ are arguably more thematically diverse than discourses entirely focused on either topic. What may set end-users apart from experts and journalists, ultimately, is the time spent contemplating the broader implications of an issue. Endusers may have a habit of homing in on one component of a problem and reflecting only on that component regardless of having been presented with all the necessary information needed to be aware of the many surrounding issues. A hypothetical example of this would be presenting endusers a news article summarizing a celebrity divorce. The outcome of one celebrity might interest end-users more than the outcome of another, and consequently, end-users may likely focus on only one celebrity when forming an opinion of the story. Present the same news article to a lawyer practising family law, and the subsequent formed opinion likely acknowledges all the possible outcomes. Although AI could very well be celebrity gossip, it is not. AI is associated with a wide range of problems that could exacerbate inequality and affect economic growth. The qualitative review of the findings echoed the quantitative observation of the data that revealed that the prevalence of the theme economy/inequality was higher for experts and journalists combined. While journalists and experts expressed dissimilar views on the direction of AI, generally both referenced the role of the economy and the consequent social implications that would result if AI became an occupational reality.

RQ2a. What sentiments guide public discourse online about the changing nature of work?

Media: The prevailing sentiments in the news media were dread and hope, both making the same amount of appearances. The subtheme neutral/mixed was the least frequent. 
Experts: The analysis of the expert quotes revealed that, like journalists, experts either felt a sense of hope or a sense of dread regarding AI and the future of work. Additionally, the subtheme neutral/mixed was the least frequent sentiment, but unlike in the news media, the neutral/mixed sentiment was slightly more frequent in expert quotes.

End-users: On Reddit, the sentiment dread dominated the discourses and essentially represented a little over half of the way end-users felt about AI and the future of work. Even when the sentiments hope and neutral/mixed were aggregated, dread was still considerably more frequent.

\section{Discussion}

Several distinctions can be made from an observation of the sentiment subthemes that emerged from the data. An equal number of experts either felt a sense of hope or dread about the future of AI in the workplace. A similar phenomenon was observed in the news media. An examination of the journalist discourses revealed that, perhaps even more so, journalists were polarized between hope and dread. There is a possible psychological explanation for these phenomena. Psychometric research on the perception of risks suggests that when assessing potentially hazardous technology, individuals do not weigh the associated risks in terms of good and bad, but rather individuals perceive technology as either good or bad based on the extent to which they see technology as socially or economically beneficial (Gregory \& Mendelsohn, 1992). The low number of neutral/mixed sentiments for experts and journalists, to this end, could be due to this tendency. Such an explanation also accounts for the low number of discourses containing the subtheme neutral/mixed from end-users.

Another finding that was observed by recording the frequencies of the sentiments was the high prevalence of dread that categorized a little over half of the discourses that came from end- 
users. This stood out considering that the data showed that experts and journalists did not tip the scales in favour of hope or dread. End-users overall were conceivably exposed to similar degrees of the sentiments hope and dread from the news media and from experts. In the realm of risk research, dread falls into the category of dread risk, which has been understood to represent spatially distant events that are perceived as unruly, catastrophic, and unjust (Slovic \& Weber, 2002; Jagiello \& Hills, 2018). This notion of dread risk similarly resembles the subtheme dread that emerged out of this MRP's inductive content analysis. In the literature, experiments have shown that the perception and social amplification of dread risk is particularly sensitive to accumulating negative information and becoming more negative over time. Dread risk even remains negative when impartial information is introduced (Jagiello \& Hills, 2018). Previous experiments, ultimately, have observed a similar phenomenon where dread becomes amplified in social discourses. The subtheme dread, like dread risk, may be particularly sensitive to social amplification. On Reddit, the social amplification of dread arguably has a greater impact on endusers as they are the individuals participating in social engagement. Experts and journalists are, unlike end-users, exclusively providing information and encouraging discussion.

RQ2b. What specific sentiments and views are the most prominent on Reddit regarding the changing nature of work and AI?

End-users: An investigation of the sentiments and views on Reddit somewhat contradicted the initial findings. A deeper investigation into the discourses of end-users on Reddit revealed that, while dread was the overall most frequent sentiment, dread was only slightly more frequent next to hope in discourses that contained the theme economy. The frequency of the sentiments dread and hope were roughly equal. When looking at the second 
most frequent theme economy/inequality, dread was exponentially more frequent than hope in the discourses of end-users. An investigation of the data on experts and journalists revealed that the subtheme hope was the predominant sentiment that appeared in their discourses that contained the themes economy and economy/inequality. In discourses with the theme economy, end-users that exhibited the hope sentiment did so because they found the views of experts and journalists to be, more or less, exaggerated. The other half of end-users that exhibited the dread sentiment did so because they perceived technological progress as an inevitable phenomenon, and they feared that AI would eventually become sophisticated enough to automate the majority of jobs.

\section{Discussion}

The findings for $\mathbf{R Q 2 b}$ showed that end-users are divided on the topic of the economy, even when they as a whole demonstrated possessing a sense of dread about AI and the future of work. This finding may appear similar to the findings in RQ2a where the data showed that experts and journalists were similarly divided between the sentiments hope and dread. The data

also showed, however, that experts and journalists were, as opposed to end-users, mostly hopeful when the topic of discussion was centred on the economy. This was also true for the theme economy/inequality; although, the subtheme hope was slightly more frequent among experts. In light of this observation, the prevailing sentiment on Reddit was dread because, even though endusers were divided between hope and dread in discourses with the theme economy, the end-users were markedly apprehensive about the future of AI compared to experts and journalists. The frequency of dread, more specifically, in economy themed discourses from end-users was high considering the modicum of dread in economy themed discourses from experts and journalists. Mentioned in the discussion for RQ2a, risk research has observed that the perception of dread 
risk is sensitive to social amplification (Jagiello \& Hills, 2018). While this notion could also apply to the findings of $\mathbf{R Q 2} \mathbf{b}$ where the subtheme dread appeared to be more impactful on endusers than journalists and experts, there is another possible explanation that may account for the unique salience of dread. The negativity bias has received considerable attention over the years and has led to the widespread belief that negative events are invariantly more salient than those events perceived to be either neutral or positive (Rozin \& Royzman, 2001). Behavioural experiments in Psychology have found that negative traits are more likely to draw attention than positive traits (Pratto \& John, 1991). At a more anatomical level, an investigation of electrical brain processes has witnessed high elevation in brainwave activity responding to negative stimuli compared with positive stimuli, further corroborating the existence of the negativity bias (Ito et al., 1998). The human tendency to exert more mental energy towards negative perceptions than positive or neutral perceptions could help to explain the high prevalence of the subtheme dread in the data overall and in the data for each theme. The reason why experts and journalists may appear to be more evenly divided could again come down to who is on the receiving end of information dissemination and social media engagement. Experts and journalists in the MRP's research were actors disseminating information. Experts and journalists, to this end, were arguably not affected by the negativity bias in the same way that end-users were because experts and journalists were the individuals providing the knowledge as opposed to learning from it.

As for the prevailing views on Reddit about AI, half of end-users felt a sense of dread regarding the economy and the ramifications that would occur as a result of AI being introduced into the workplace. On the other hand, half of end-users felt hopeful about the future of AI in the context of work and the economy. End-users that were hopeful about the future of work tended to perceive the current economic infrastructure as an arbitrator that precluded mass automation. 
End-users that felt a sense of dread about the future of work perceived the contrary. Whether that meant fruitless reskilling or technological unemployment, the economy was deemed ill-equipped to deal with advanced AI in the workplace. While the data showed that hope and dread were essentially equally frequent, the dread subtheme did outnumber the hope subtheme by one. RQ2b was developed largely in part to identify public opinion on Reddit. The overview of public opinion provided in the literature conceptualized the term as the aggregation of collective sentiment (Herbts, 1993). In view of this notion, the aggregation of collective sentiment in the theme economy dictates that, due to a slight majority, a sense of dread about AI and the economy is representative of public opinion on Reddit. This definition of public opinion also applies to the high prevalence of dread in the other two themes respectively. The views that culminate public opinion on Reddit ultimately reflect the perception that the introduction of AI into the workplace will hurt the economy, lead to greater inequality, or both. 


\section{Conclusion}

Technology has continued to evolve and create new opportunities all the while changing others. Mechanization and AI have essentially become synonymous in the current epoch as new developments and advancements in technology enable AI to take on occupational roles that have been traditionally held by human workers. This MRP, for this reason, was interested in understanding and demystifying the current zeitgeist surrounding an area of technology that is both inflammatory and propitious. Using the social news site Reddit, this MRP investigated online public opinion by collecting data from comments, media articles, and expert quotes. Experts and lay people, in addition, generally have different perspectives on different issues, and the news media is often a tool that bridges the thoughts and feelings of experts with the thoughts and feelings of everyday, ordinary people.

As realm of deliberation, Reddit provides a platform where opinions can be formed, and due to Reddit's design, individuals have access to an arena where they can discuss and deliberate over topics that are of most interest to them. Having completed an analysis of the data, the research concluded with several findings. Firstly, experts and journalists, more often than not, shared similar conceptions and sentiments about AI and the future of work. Either hopeful or overall divided, experts and journalists demonstrated conspicuous parallels. Secondly, end-users differed from journalists and experts in that end-users spoke mostly about the economy and exhibited negative feelings about it. Lastly, end-users on Reddit demonstrated that altogether they dreaded the introduction of AI into the workplace and the consequent future of work where AI would play a pivotal role in a new occupational reality. 


\section{Further Study}

This MRP contributes to various bodies of research ranging from risk communication to public policy. Observing public deliberation on social media as well as highlighting the perceptions end-users exhibited towards potentially hazardous technology, the research demonstrated some of the ways news can be disseminated online and potentially guide any given topic towards any given sentiment. Possible directions for further study include, but are not limited to, investigating the direct impact of expert consensus on public opinion in relation to the introduction of controversial technology, or examining in an offline context the prevailing views and sentiments of lay people regarding AI and the future of work. 


\section{Appendix A}

Codebook of Themes Identified from Inductive Content Analysis

\begin{tabular}{|c|c|c|}
\hline Theme & Description & Example \\
\hline Economy & $\begin{array}{l}\text { Depicts the economy as the } \\
\text { primary issue in the text. AI } \\
\text { presents or alleviates } \\
\text { obstacles to employment or } \\
\text { other facets of the economy. }\end{array}$ & $\begin{array}{l}\text { "Well yeah wages are } \\
\text { currently a balance between } \\
\text { labor and capital. The less } \\
\text { that our labor is require[d] } \\
\text { the more wealth will } \\
\text { centralise. But I doubt you } \\
\text { could tax the manufacturers } \\
\text { enough to support a welfare } \\
\text { state." }\end{array}$ \\
\hline Inequality & $\begin{array}{l}\text { Depicts inequality as the } \\
\text { primary issue in the text. } \\
\text { Discourses foreground a } \\
\text { dichotomy between different } \\
\text { groups of people and the } \\
\text { challenges or positive } \\
\text { changes that AI will bring } \\
\text { into society. }\end{array}$ & $\begin{array}{l}\text { "[I] bet there will be a tax } \\
\text { on the robots - but it will be } \\
\text { the wrong kind of tax, the } \\
\text { kind that keeps regular joe } \\
\text { from owning them and puts } \\
\text { them exclusively in the } \\
\text { hands of the corporations." }\end{array}$ \\
\hline Economy/Inequality & $\begin{array}{l}\text { Depicts both major themes. } \\
\text { Messages portray AI as a } \\
\text { technology with both societal } \\
\text { and economic implications. }\end{array}$ & $\begin{array}{l}\text { "This is not an upside at all } \\
\text { if you consider the fact that } \\
\text { society actually progresses } \\
\text { and theoretically wouldn't } \\
\text { have to work at all if the } \\
\text { profits made through } \\
\text { technological revolution } \\
\text { were distributed to the } \\
\text { population." }\end{array}$ \\
\hline
\end{tabular}




\section{Appendix B}

Codebook of Subthemes Identified from Inductive Content Analysis

\begin{tabular}{|c|c|c|}
\hline Subtheme & Description & Example \\
\hline Hope & $\begin{array}{l}\text { Hopeful about the future of } \\
\text { AI in the context of one of } \\
\text { the major themes. }\end{array}$ & $\begin{array}{l}\text { "You will always need } \\
\text { people to fix the machines } \\
\text { and who understand them. } \\
\text { You will also need people to } \\
\text { design and build the new } \\
\text { machines. Engineering and } \\
\text { technology education will } \\
\text { be in high demand for } \\
\text { generations to come." }\end{array}$ \\
\hline Neutral/Mixed & $\begin{array}{l}\text { Indifferent attitude or mixed } \\
\text { feelings about the future of } \\
\text { AI in the context of one of } \\
\text { the major themes. }\end{array}$ & $\begin{array}{l}\text { "Yes, now it's all 'service } \\
\text { economy make-work.' Bring } \\
\text { on the telephone sanitizers I } \\
\text { guess. We've had more } \\
\text { humans than we have had } \\
\text { work for humans for a long } \\
\text { time now, and nobody really } \\
\text { has an answer (that isn't a } \\
\text { fundamental re-work of how } \\
\text { human beings view other } \\
\text { people, assets, money, etc.)" }\end{array}$ \\
\hline Dread & $\begin{array}{l}\text { A sense of dread towards the } \\
\text { future of AI in the context of } \\
\text { one of the major themes. }\end{array}$ & $\begin{array}{l}\text { "What makes me really } \\
\text { nervous, I don't believe in } \\
\text { some sort of jobless utopia. } \\
\text { Rich people don't work like } \\
\text { that. There's no such thing } \\
\text { as enough for them. They'll } \\
\text { give the } 50 \% \text { who lose their } \\
\text { jobs to automation enough } \\
\text { to keep living at a } \\
\text { subsistence level. } \\
\text { If there are riots, guess what } \\
\text { happens? That's when } \\
\text { machines start using guns." }\end{array}$ \\
\hline
\end{tabular}




\section{References}

Acemoglu, D., \& Restrepo, P. (2018). Artificial intelligence, automation and work (NBER Working Paper 24196). Cambridge, MA: National Bureau of Economic Research. Retrieved from the National Bureau of Economic Research: https://www.nber.org/papers/w24196

Aiva. (2017, December 21). Making of Pixelfield "Battle Royale" - The world's first AIcomposed score for a video game [Video]. YouTube. https://www.youtube.com/watch?v=6k-7yxNF3UM

Anstead, N., \& O’Loughlin, B. (2015). Social media analysis and public opinion: The 2010 UK general election. Journal of Computer-Mediated Communication, 20(2), 204220. https://doi.org/10.1111/jcc4.12102

Autor, D. H. (2013) The "task approach" to labor markets: An overview. Journal for Labour Market Research, 46, 185-199. https://doi-org.ezproxy.lib.ryerson.ca/10.1007/s12651$\underline{013-0128-Z}$

Autor, D. H. (2015). Why are there still so many jobs? The history and future of workplace automation. The Journal of Economic Perspectives, 29(3), 3-30. Www.jstor.org/stable/43550118

Barreau, P. (2018, April). How AI could compose a personalized soundtrack to your life [Video]. TED. https://www.ted.com/talks/pierre_barreau_how_ai_could_compose_a personalized_soun dtrack to your_life\#t-594

Bengtsson, M. (2016). How to plan and perform a qualitative study using content analysis. NursingPlus Open, 2, 8-14. https://doi.org/10.1016/j.npls.2016.01.001 
Bessen, J. (2018). Automation and jobs: When technology boosts employment. Economic Policy. https://doi.org/10.1093/epolic/eiaa001

Boukes, M. (2019). Social network sites and acquiring current affairs knowledge: The impact of Twitter and Facebook usage on learning about the news. Journal of Information Technology \& Politics, 16(1), 36-51. https://doi.org/10.1080/19331681.2019.1572568

Borenstein, J. (2009). Robots and the changing workforce. AI \& SOCIETY, 26(1), 87-93. https://doi.org/10.1007/s00146-009-0227-0

Borland, J., \& Coelli, M. (2017). Are robots taking our jobs? Australian Economic Review, 50(4), 377-397. https://doi.org/10.1111/1467-8462.12245

Bruininks, P., \& Malle, B. F. (2005). Distinguishing hope from optimism and related affective states. Motivation and Emotion, 29, 324-352. https://doi.org/10.1007/s11031-006-9010-4

Chah, N. (2019). Down the deep rabbit hole: Untangling deep learning from machine learning and artificial intelligence. First Monday, 24(2). https://doi.org/10.5210/fm.v24i2.8237

Churcher, P. R. (1991). The impact of artificial intelligence on leisure. AI \& Society, 5(2), 147155. https://doi.org/10.1007/BF01891720

Collins, H. M., \& Evans, R. (2002). The third wave of science studies: Studies of expertise and experience. Social Studies of Science, 32(2), 235296. https://doi.org/10.1177/0306312702032002003

Craft, G. A. (1992). The persistence of dread in law and literature. The Yale Law Journal, 102(2), 521-546. https://doi.org/10.2307/796846

Deuze, M. (2001). Online journalism: Modelling the first generation of news media on the World Wide Web. First Monday, 6(10). https://doi.org/10.5210/fm.v6i10.893

Dockterman, E. (2019, November 1). Untangling the Terminator franchise's complicated 
timeline. Time. https://time.com/5697301/terminator-movies-timeline-explained/

E1. (2018, April 21). So late to the party, damn time zones [Comment on the online forum post Artificial intelligence will wipe out half the banking jobs in a decade, experts say]. Reddit.

https://www.reddit.com/r/technology/comments/8drci8/artificial_intelligence_will_wipe out_half_the/dxq5ilh/

E2. (2016, November 11). I'm going into the tourism industry [Comment on the online forum post 224 global investors with more than $\$ 100$ billion of investable capital say that governments are not prepared for the coming of artificial intelligence and the massive job loss that will result.]. Reddit.

https://www.reddit.com/r/worldnews/comments/5c9lc8/224_global_investors_with_more than 100 billion/d9vpv2w/

E3. (2017, March 7). This analysis just don't hold water. [Comment on subreddit $\mathrm{r} /$ Futurology on the online forum post Robots won't just take our jobs - they'll make the rich even richer: "Robotics and artificial intelligence will continue to improve - but without political change such as a tax, the outcome will range from bad to apocalyptic"]. Reddit.

https://www.reddit.com/r/Futurology/comments/5x2pw4/robots_wont just take_our_job s theyll_make the/deexj2e/

E4. (2016, November 11). Let's break it down here, how many positions are going to be wiped out by AI once they become mainstream? [Comment on the online forum post 224 global investors with more than $\$ 100$ billion of investable capital say that governments are not prepared for the coming of artificial intelligence and the massive 
job loss that will result.]. Reddit.

https://www.reddit.com/r/worldnews/comments/5c9lc8/224_global_investors_with_more than 100 billion/d9vtlud/

Fama, E. F. (1970). Efficient capital markets: A review of theory and empirical work. The Journal of Finance, 25(2), 383-417. https://doi.org/10.2307/2325486

Frey, C. B., \& Osborne, M. A. (2017). The future of employment: How susceptible are jobs to computerisation? Technological Forecasting \& Social Change, 114, 254-280. https://doi.org/10.1016/j.techfore.2016.08.019

Gil de Zúñiga, H., Jung, N., \& Valenzuela, S. (2012). Social media use for news and individuals' social capital, civic engagement and political participation. Journal of ComputerMediated Communication, 17(3), 319-336. https://doi.org/10.1111/j.1083$\underline{6101.2012 .01574 . x}$

Graneheim, U. H., Lindgren, B.-M., \& Lundman, B. (2017). Methodological challenges in qualitative content analysis: A discussion paper. Nurse Education Today, 56, 2934. https://doi.org/10.1016/j.nedt.2017.06.002

Gregory, R., \& Mendelsohn, R. (1993). Perceived risk, dread, and benefits. Risk Analysis, 13(3), 259-264. https://doi.org/10.1111/j.1539-6924.1993.tb01077.x

Herbst, S. (1993). The meaning of public opinion: Citizens' constructions of political reality. Media, Culture \& Society, 15(3), 437-

454. https://doi.org/10.1177/016344393015003007

Hsieh, H.-F., \& Shannon, S. E. (2005). Three approaches to qualitative Content Analysis. Qualitative Health Research, 15(9), 12771288. https://doi.org/10.1177/1049732305276687 
Ito, T. A., Larsen, J. T., Smith, N. K., \& Cacioppo, J. T. (1998). Negative information weighs more heavily on the brain: The negativity bias in evaluative categorizations. Journal of Personality and Social Psychology, 75(4), 887-900. https://doi.org/10.1037/0022$\underline{3514.75 .4 .887}$

Jagiello, R. D., \& Hills, T. T. (2018). Bad news has wings: Dread risk mediates social amplification in risk communication. Risk Analysis, 38(10), 21932207. https://doi.org/10.1111/risa.13117

Keynes, M. J. (1930). Economic possibilities for our grandchildren [Scanned from Essays in Persuasion]. http://www.econ.yale.edu/smith/econ116a/keynes1.pdf

Kalra, A., Singh, S., Singh, S., Singh, S., Singh, G., \& Thappa, M. (2010). Artificial intelligence and neural network. International Journal of Advanced Research in Computer Science, 1(3). http://ezproxy.lib.ryerson.ca/login?url=https://search-proquestcom.ezproxy.lib.ryerson.ca/docview/1443702162?accountid=13631

Kaplan, A. M., \& Haenlein, M. (2010). Users of the world, unite! The challenges and opportunities of Social Media. Business Horizons, 53(1), 5968. https://doi.org/10.1016/j.bushor.2009.09.003

Koetsier, J. (2016, November 10). 93\% of investors say AI will destroy jobs, governments not prepared. Forbes. https://www.forbes.com/sites/johnkoetsier/2016/11/10/93-of-investorssay-ai-will-destroy-jobs-governments-not-prepared/

Lamb, C. (2016). The talented Mr. Robot: The impact of automation on Canada's workforce. Brookfield Institute for Innovation and Entrepreneurship. http://brookfieldinstitute.ca/report/the-talented-mr-robot/

McClelland, C. (2017, December 4). The difference between artificial intelligence, machine 
learning, and deep learning. Medium. https://medium.com/iotforall/the-differencebetween-artificial-intelligence-machine-learning-and-deep-learning-3aa67bff5991

McKendrick, J. (2018, January 25). Artificial intelligence isn't killing jobs; It's killing business models. Forbes. https://www.forbes.com/sites/joemckendrick/2018/01/25/artificialintelligence-isnt-killing-jobs-its-killing-business-models/

Morse, J. M. (2008). Confusing categories and themes. Qualitative Health Research, 18(6), 727728. https://doi.org/10.1177/1049732308314930

Murphy, N., (2019, December 4). Reddit's 2019 year in review.

Upvoted. https://redditblog.com/2019/12/04/reddits-2019-year-in-review/

OECD. (2019). OECD employment outlook 2019: The future of work. https://doi.org/10.1787/9ee00155-en

Page, B. I., Shapiro, R. Y., \& Dempsey, G. R. (1987). What moves public opinion? American Political Science Review, 81(1), 23-43. https://doi.org/10.2307/1960777

Pratto, F., \& John, O. P. (1991). Automatic vigilance: The attention-grabbing power of negative social information. Journal of Personality and Social Psychology, 61(3), 380391. https://doi.org/10.1037/0022-3514.61.3.380

Rosenbaum, E. (2018, March 23). Facebook data privacy scandal has one silver lining: Thousands of new jobs AI can't handle. CNBC. https://www.cnbc.com/2018/03/23/facebook-privacy-scandal-has-a-plusthousands-of-new-jobs-ai-cant-do.html

Rozin, P., \& Royzman, E. B. (2001). Negativity bias, negativity dominance, and contagion. Personality and Social Psychology Review, 5(4), 296320. https://doi.org/10.1207/S15327957PSPR0504_2 
Schwab, K. (2016, January 12). The fourth industrial revolution: what it means, how to respond. World Economic Forum. https://www.weforum.org/agenda/2016/01/the-fourthindustrial-revolution-what-it-means-and-how-to-respond/

Shearer, E. (2018, December 10). Social media outpaces print newspapers in the U.S. as a news source. Pew Research Center. https://www.pewresearch.org/fact-tank/2018/12/10/socialmedia-outpaces-print-newspapers-in-the-u-s-as-a-news-source/

Shepard, W. J. (1909). Public opinion. American Journal of Sociology, 15(1), 32-60. www.jstor.org/stable/2762619

Siegrist, M., Keller, C., Kastenholz, H., Frey, S., \& Wiek, A. (2007). Laypeople’s and experts’ perception of nanotechnology hazards. Risk Analysis, 27(1), 5969. https://doi.org/10.1111/j.1539-6924.2006.00859.x

Simmons, A. B., \& Chappell, S. G. (1988). Artificial intelligence-definition and practice. IEEE Journal of Oceanic Engineering, 13(2), 14-42. https://doi.org/10.1109/48.551

Slovic, P., \& Weber, E. U. (2002, April 12-13). Perception of risk posed by extreme events [Prepared for conference]. Risk Management strategies in an Uncertain World, Palisades, New York, United States. https://www.aaai.org/ocs/index.php/ICWSM/ICWSM16/paper/view/13154/12766

Syam, N., \& Sharma, A. (2018). Waiting for a sales renaissance in the fourth industrial revolution: Machine learning and artificial intelligence in sales research and practice. Industrial Marketing Management, 69, 135-146. https://doi.org/10.1016/j.indmarman.2017.12.019

Tarnoff, B. (2017, March 2). Robots won't just take our jobs-They'll make the rich even 
richer. The Guardian. https://www.theguardian.com/technology/2017/mar/02/robot-taxjob-elimination-livable-wage

van Heek, J., Arning, K., \& Ziefle, M. (2017). Differences between laypersons and experts in perceptions and acceptance of CO2-utilization for plastics production. Energy Procedia, 114, 7212-7223. https://doi.org/10.1016/j.egypro.2017.03.1829

Weninger, T. (2014). An exploration of submissions and discussions in social news: Mining collective intelligence of Reddit. Social Network Analysis and Mining, 4, 173. https://doi.org/10.1007/s13278-014-0173-9

Zafar, M., Gummadi, K., \& Danescu-Niculescu-Mizil, C. (2016). Message impartiality in social media discussions. Proceedings of the Tenth International AAAI Conference on Web and Social Media. https://www.aaai.org/ocs/index.php/ICWSM/ICWSM16/paper/view/13154/12766 